\title{
SOME SUFFICIENT CONDITIONS FOR THE UNIVALENCE OF AN INTEGRAL OPERATOR
}

\author{
Halit ORHAN, Dorina RÃduCANu AND Murat ÇAĞLAR
}

Abstract. Making use of the method of subordination chains, we obtain some sufficient conditions for the univalence of an integral operator. In particular, as special cases, our results imply certain known univalence criteria. A refinement to a quasiconformal extension criterion of the main result, is also obtained.

Mathematics subject classification (2010): Primary 30C80; Secondary 30C45, 30 C62. sion.

Keywords and phrases: Loewner chain, univalent function, integral operator, quasiconformal exten-

\section{REFERENCES}

[1] L. V. Ahlfors, Sufficient conditions for quasiconformal extension, Ann. Math. Studies. 79 (1974), 23-29.

[2] J. M. Anderson, A. HinkKanen, Univalence criteria and quasiconformal extensions, Trans. Amer. Math. Soc. 324 (1991), 823-842.

[3] J. BECKER, Löwnersche differential Gleichung und quasikonform fortsetzbare schlichte Funktionen, J. Reine Angew. Math. 255 (1972), 23-43.

[4] J. BECKER, Über die Lösungsstruktur einer Differentialgleichung in der Konformen Abbildung, J. Reine Angew. Math. 285 (1976), 66-74.

[5] J. BECKER, Conformal mappings with quasiconformal extensions, Aspects of Contemporary Complex Analysis, Ed. by D. A. Brannan and J. G. Clunie, Acad. Press, 1980, 37-77.

[6] Th. BetKer, Löewner chains and quasiconformal extensions, Complex Variables Theory Appl. 20, 1-4 (1992), 107-111.

[7] N. Danikas, S. Ruscheweyh, Semi-convex hulls of analytic functions in the unit disk, Analysis 19, 4 (1999), 309-318.

[8] E. Deniz, H. Orhan, Some notes on extensions of basic univalence criteria, J. Korean Math. Soc. 48, 1 (2011), 179-189.

[9] I. HotTA, Löwner chains with complex leading coefficient, Monatsh Math. 163 (2011), 315-325.

[10] I. HоттA, Explicit quasiconformal extensions and Loewner chains, Proc. Japan Acad. Ser. A. 85 (2009), 108-111.

[11] I. HотTA, Löewner chains and quasiconformal extension of univalent functions, Dissertation, Tohoku Univ., 2010.

[12] J. G. KRÝZ, Convolution and quasiconformal extension, Comm. Math. Helv. 51 (1976), 99-104.

[13] S. Kanas And H. M. SRIVAstava, Some criteria for univalence related to Ruscheweyh and Salagean derivatives, Complex Var. Elliptic Equ. 38 (1997), 263-275.

[14] Z. Lewandowski, On a univalence criterion, Bull. Acad. Polon. Sci. Ser. Sci. Math. 29 (1981), $123-126$.

[15] S. Moldoveanu, On an univalence condition, Seminar on Geometric Functions Theory (Preprint), 3 (1993), 49-54.

[16] H. Ovesea, An extension of Lewandowski's univalence criteria, Demonstratio Mathematica (Warszawa) XXIX, 4 (1996), 699-706.

[17] H. OvesEA, A generalization of Ruscheweyh's univalence criterion, J. Math. Anal. Appl. 258 (2001), $102-109$. 
[18] H. Ovesea, N. N. Pascu, I. Radomir, On a univalence criterion, Mathematica (Cluj-Napoca) 36(59), 2 (1994), 209-214.

[19] N. N. PASCU, Sufficient conditions for univalence, Seminar on Geometric Functions Theory (Preprint), 5 (1986), 119-122.

[20] N. N. PASCU, An improvement of Becker's univalence criterion, Commemorative Session Simion Stoilov, Univ. of Braşov (Preprint), 1987, 43-48.

[21] J. A. PfaltzgrafF, K-quasiconformal extension criteria in the disk, Complex Variables 21 (1993), 293-301.

[22] Ch. Pommerenke, Über die Subordination analytischer Funktionen, J. Reine Angew Math. 218 (1965), 159-173.

[23] Ch. Pommerenke, Univalent Functions, Vandenhoeck Ruprecht in Göttingen, 1975.

[24] D. Rãducanu, H. Orhan, E. Deniz, On some sufficient conditions for univalence, An. Şt. Univ. "Ovidius" Constanta Ser. Mat. 18, 2 (2010), 217-222.

[25] St. Ruscheweyh, An extension of Becker's univalence condition, Math. Ann. 220 (1976), 285-290. 